\title{
High spin particles with spin-mass coupling II
}

\author{
M. DASZKIEWICZ \\ Institute of Theoretical Physics, Wrocław University, pl. Maxa Borna 9 \\ 50-206 Wrocław, Poland \\ marcin@ift.uni.wroc.pl \\ Z. HASIEWICZ, T. NIKICIUK and C. J. WALCZYK \\ Institute of Theoretical Physics, University of Białystok, ul. Lipowa 41 \\ 15-424 Białystok, Poland \\ zhas@uwb.edu.pl, niki@alpha.uwb.edu.pl, c.walczyk@alpha.uwb.edu.pl
}

\begin{abstract}
The classical and quantum model of high spin particles within the manifestly covariant framework. The internal (spin) degrees of freedom are described by two $\mathcal{C}(3,1)$ Clifford algebra spinors. The covariant quantization leads to PCT invariant spectrum of particles with spin dependent masses. The quantum model contains elementary particles and the cluster states generating infinite degeneracy of the mass spectrum.
\end{abstract}

\section{Introduction}

There are many possible descriptions of the spinning particles with arbitrary spin spectrum. It seems, that the most promising ones are so called spinorial models [1]-6] with the internal degrees of freedom (spin) realized by classical, (anti-)commuting spinors, i.e the elements of irreducible or reducible representation modules of corresponding Clifford algebras, most commonly $\mathcal{C}(3,1)$. Despite of the fact that their construction relies on direct generalization of superparticle models they contain the particles of arbitrarily high spins. However, the masses of these particles appear to be the same once the value of the mass parameter of the model is fixed. There are also constructions [7] which lead to spin dependent masses of the particles but, in contrast to the models mentioned above, with spin variable introduced as a free parameter.

From this point of view the classical model defined in [8] and analyzed in details in [9] seems to be particulary interesting. It's construction relies on minimal coupling of the particle trajectory with vector current build up of single $\mathcal{C}(3,1)$ real (Majorana) spinor. Due to this coupling the resulting first quantized model describes the infinite families of spinning particles with spin-dependent masses. As the analysis at the classical level strongly indicates this is this very coupling, which corresponds to, and is responsible for spinning particles "zitterbewegung" phenomenon [11], [12],[13], which is most clearly and efficiently described in Clifford algebra language.

Besides of the "dynamical" spin-mass coupling, the spinning particle model of [8] and [9] has an additional advantage: the non degenerate mass levels. Unfortunately, it has also important drawback. The first quantization results in the Hilbert space of states, which contains orphaned particles and antiparticles, i.e. it breaks CPT symmetry

\footnotetext{
${ }^{1}$ The corresponding antiparticles are missing.
} 
[10]. Consequently, it does not allow one to construct the corresponding local quantum field theory by direct application of the second quantization procedures [10].

The main task of this paper is to present the extended spinorial model which cures the above weakness of the prototype proposed in [9]. The appropriate modification consists in adding an additional spinorial degree of freedom with opposite spin-mass coupling constant. The model obtained in this straightforward way contains however the non-linear interaction of spinors. This interaction is eliminated by the substraction from the Lagrange function the quartic term in spinorial variables (see (41)). As a result, one gets after first quantization, the infinitly degenerate but CPT invariant spectrum of particles.

As in the case of [9] the paper contains also covariant formulation of the model based on the complex polarization of the second class constraints and application of GuptaBleuler quantization procedure [14]. The polarization leads to the description of the internal degrees of freedom in terms of Weyl variables i.e. in the language of even subalgebra $\mathcal{C}(3,1)_{0} \approx \mathcal{C}(3,0)$ spinors. This approach results in a natural generalization of Dirac type equations [15] for the particles with "dynamical" spin-dependent mass.

Since the above model has CPT invariant mass spectrum, it can serve as a starting point for the construction of the local quantum field theory [10] for particles lying on Regge trajectory. It would allow one, in particular, to investigate the different kinds of field theoretical interactions of the spinning particles with arbitrary spin and with spin dependent mass in four dimensional space-time.

As far as we know, the quantum field theoretical description of the particles lying on Regge trajectories was proposed many years ago in the series of papers [17, [18, [19], [20]. Apart of that, such a description was also elaborated in the framework of dual model [21] and string theory [22], 23], but only for the critical dimension of space-time. In the case of dimension four, the direct constructions of this kind encounter serious difficulties and problems related to the presence of Liouville modes [24].

The paper is organized as follows.

In the first chapter the structure of the classical model is presented in Lagrangian and Hamiltonian form. The complex parametrization of the spinor degrees of freedom in terms of Weyl spinors is introduced in order to make the transparent link with the commonly used formalism.

The next chapter is devoted to the description of particle content of the corresponding quantum theory. The spectrum is divided into two series: the elementary trajectories and the sector describing clusters.

Finally, the results are summarized and some open questions and problems are raised.

\section{The classical model}

The classical model considered in this paper is a natural generalization and extension of the one presented in [9]. The particle content of the model of [9] appeared to be not CPT invariant: the particles and antiparticles were orphaned.

From the form of the mass spectrum obtained in [9]: $m_{j}=\sqrt{h^{2} j^{2}+m_{0}^{2}}-h j$ for 
the particles of spin $j$ and $m_{j}=\sqrt{h^{2} j^{2}+m_{0}^{2}}+h j$ for antiparticles, one may easily conclude that in order to get rid of this undesired orphancy, it is enough to add an additional spinorial degree of freedom with opposite spin-mass coupling. The classical model obtained in such a way was already proposed in the Conclusions of [9]. It is defined by the following Lagrange function:

$$
\mathcal{L}_{0}=\frac{1}{2} e^{-1} \dot{x}^{2}-\frac{1}{2} e m_{0}^{2}+\frac{h}{2} \dot{x} \cdot j_{1}-\frac{h}{2} \dot{x} \cdot j_{2}+\bar{\eta}_{1} \dot{\eta}_{1}+\bar{\eta}_{2} \dot{\eta}_{2}
$$

which is a straightforward extension of the one presented in [9]. In addition to the standard terms describing the scalar particle of mass $m_{0}$ it contains "minimal" couplings of the velocity with two spinor currents

$$
j_{I}^{\mu}=\bar{\eta}_{I} \gamma^{\mu} \eta_{I}, I=1,2
$$

The currents are build out of two independent Majorana spinors $\eta_{I}$. For the Majorana spinors to exist one should assume the spacetime metric in the form $g=$ $\operatorname{diag}(-1,+1,+1,+1)$ i.e. the Clifford algebra generators present in (2) should satisfy:

$$
\gamma^{\mu} \gamma^{\nu}+\gamma^{\nu} \gamma^{\mu}=2 g^{\mu \nu}
$$

The spinorial conjugation is defined in the standard way: $\bar{\eta}_{I}=\eta_{I}^{\mathrm{T}} \gamma_{0}$ and it defines the $\operatorname{Spin}(3,1)$ invariant scalar product: $\bar{\eta} \eta^{\prime}=\eta_{I}^{\mathrm{T}} \gamma_{0} \eta^{\prime}$. The explicite form of this product is not in fact needed. The only property which is used further on is that it generates $\beta_{-}$antiautomorphism of $\mathcal{C}(3,1)$ Clifford algebra: $\beta_{-}\left(\gamma^{\mu}\right)=-\gamma^{\mu}$.

The classical model defined by (11) appears to generate non-linear classical equations of motion.

Besides of the standard algebraic equation or 1-bein variable $e$ and momentum conservation law:

$$
\frac{d}{d \tau} p^{\mu}=0, \quad \text { where } \quad p^{\mu}=\frac{1}{e} \dot{x}^{\mu}+\frac{h}{2} j_{1}^{\mu}-\frac{h}{2} j_{2}^{\mu},
$$

it contains non-linear equations of motion for spinor variables:

$$
\frac{d}{d \tau} \eta_{1 / 2}=\frac{e h}{2}\left(\mp p^{\mu}-\frac{h}{2} j_{2 / 1}^{\mu}\right) \gamma_{\mu} \eta_{1 / 2}
$$

The interactions of this type are absent in single spinor model of [9] as the spin vector currents built out of single $\mathcal{C}(3,1)$ Clifford algebra spinors are light-like. This fact can be easily proved by the use of Clifford algebra structural relations without any use of explicit matrix representation. This non-trivial "interaction" of spinors follows from the fact that both spinors are coupled to single particle trajectory.

For this reason the model defined by (1) is difficult to treat technically at both: classical and quantum levels.

The form of the equations of motion (3) strongly suggests that the interaction of spinors is of potential character. It is then sensible to ask for the corresponding linearized, free model. It appears that it can be obtained by adding to (1) the term which describes the cross interaction of spinor currents:

$$
\mathcal{L}_{\text {int }}=-\frac{e h^{2}}{4} j_{1} \cdot j_{2} .
$$


It is not difficult to check (using the property of spinor currents $j_{I}^{2}=0$ being null and Schwartz inequality) that (4) is non-negative. This means that the interaction present in (11) and in (3) is governed by non-negative potential of fourth order. As it appears the term (4) cancels the non-linear interactions at the Hamiltonian level and one is left with relatively simple system of constraints of mixed type. On the other hand its presence leads to essential modification of the proper time parameter. As it can be calculated from the equations of motion for 1-bein $e$, the corresponding density gets rescaled:

$$
d s=\sqrt{-\dot{x}^{2}} d \tau \rightarrow d s^{\prime}=\sqrt{1+\frac{h^{2} j_{1} \cdot j_{2}}{2 m_{0}^{2}}} \sqrt{-\dot{x}^{2}} d \tau .
$$

The questions related to the above change of the internal geometry of the particle trajectory are by all means interesting, but they will be not pursued here. The considerations of this paper will be focused on the hamiltonian formulation and the corresponding first quantized theory.

At the canonical level the model defined by the sum of (11) and (4) describes the constrained system with constraints of mixed type. Their structure is analogous to that of $[9]$.

There are two families of spinorial constraints:

$$
G_{I}^{\alpha}=\pi_{I}^{\alpha}+\eta_{I}^{\alpha} ; \alpha=1, \ldots, 4 ; I=1,2,
$$

relating the canonical momenta $\pi_{I}^{\alpha}$ of spinors with spinors $\eta_{I}^{\alpha}$ themselves 2 .

These constraints reflect the property of the system defined by (11) and (4) that the equations of motion for spinors are of first order in the evolution parameter. They are obviously of second class:

$$
\left\{G_{I}^{\alpha}, G_{J}^{\beta}\right\}=2 \delta_{I J} C^{\alpha \beta}
$$

There is in addition the kinematic constraint related to the reparametrization invariance of the corresponding action functional. It is given by canonical Dirac hamiltonian:

$$
H_{\mathrm{D}}=\frac{1}{2}\left(p^{2}+m_{0}^{2}\right)-\sum_{I=1}^{2} \frac{h}{2}(-1)^{I} p_{\mu} J_{I}^{\mu}
$$

where $p_{\mu}$ are the momenta canonically conjugated to the positions $x^{\mu}$. The currents $J_{I}^{\mu}$ are bilinear in spinors and their conjugated momenta: $J_{I}^{\mu}=\pi_{I}^{\alpha}\left(\gamma^{\mu}\right)_{\alpha \beta} \eta_{I}^{\beta}$. The 1-bein variable was eliminated by putting $e=1$.

The constraints (6) together with (8) constitute the closed system of mixed type as one has:

$$
\left\{H_{\mathrm{D}}, G_{I}^{\alpha}\right\}=(-1)^{I} \frac{h}{2} p_{\beta}^{\alpha} G_{I}^{\beta}
$$

It should be stressed that the expression (8) and the relation (9) are so simple exactly due to the substraction of the fourth order term (4) $\sim j_{1} \cdot j_{2}$ from the Lagrange function (11).

\footnotetext{
${ }^{2}$ One obviously assumes the canonical Poisson brackets $\left\{\pi_{I}^{\alpha}, \eta_{J}^{\beta}\right\}=\delta_{I J} C^{\alpha \beta}$. The matrix $C^{\alpha \beta}$ is the inverse of the one defined in the following way: $\bar{\eta}_{I} \eta_{I}^{\prime}=\eta_{I}^{\mathrm{T}} \gamma_{0} \eta_{I}^{\prime}=\eta_{I}^{\alpha} C_{\alpha \beta} \eta_{I}^{\prime \beta}$.
} 
As it was noted in [9] the system of constraints (6) and (9) can be replaced by an equivalent system of first class by polarization of (17) [14]. For massive momenta $\left(p^{2}<0\right)$ the polarization of constraints is necessarily complex.

For this reason the system is most conveniently described in terms of minimal building blocks of $\mathbf{S p i n}(3,1) \approx \mathbf{S L}(2 ; \mathbb{C})$ representations i.e. in terms of complex Weyl spinors. The real space of Majorana spinors $\left(\eta_{I}^{\alpha}, \pi_{I}^{\alpha}\right)$ decomposes into, mutually complex conjugated ${ }^{3}$, Weyl components $\left(z_{I}^{A}, \mathfrak{z}_{I}^{A}\right)_{A=1,2}$ and $\left(z_{I}^{\bar{A}}, \mathfrak{z}_{I}^{\bar{A}}\right)_{\bar{A}=1,2}$. They span the eigensubspaces of $\gamma^{5}$ matrix corresponding to $\pm i$ eigenvalues.

The Poisson brackets of the canonical Weyl variables are given as follows:

$$
\left\{\mathfrak{z}_{I}^{A}, z_{J}^{B}\right\}=\epsilon^{A B} \delta_{I J}, \quad\left\{\mathfrak{z}_{I}^{\bar{A}}, z_{J}^{\bar{B}}\right\}=\epsilon^{\bar{A} \bar{B}} \delta_{I J},
$$

where $\epsilon^{A B}\left(\epsilon^{\bar{A} \bar{B}}\right)$ are $\mathbf{S L}(2 ; \mathbb{C})$ invariant tensors.

The second class constraints of (6) relate the canonical Weyl coordinates: $G_{I}^{A}=\mathfrak{z}_{I}^{A}+$ $z_{I}^{A}=0$ and $G_{I}^{\bar{A}}=\mathfrak{z}_{I}^{\bar{A}}+z_{I}^{\bar{A}}=0$. Their polarized counterparts can be expressed in the following way:

$$
G_{I( \pm)}^{A}=p_{\bar{B}}^{A} G_{I}^{\bar{B}} \pm i m(p) G_{I}^{A}, G_{I( \pm)}^{\bar{A}}=p_{B}^{\bar{A}} G_{I}^{B} \pm i m(p) G_{I}^{\bar{A}}
$$

where $p_{\bar{B}}^{A}$ and $p_{B}^{\bar{A}}$ are (mutually complex adjoint) matrix elements of the real operator $p^{\mu} \gamma_{\mu}$ in the complex basis of Weyl spinors. Due to Clifford algebra relations they do satisfy: $p_{\bar{B}}^{A} p_{C}^{\bar{B}}=p^{2} \delta_{C}^{A}$ and $p_{B}^{\bar{A}} p_{\bar{C}}^{B}=p^{2} \delta_{\bar{C}}^{\bar{A}}$.

The Hamiltonian constraint rewritten in terms of Weyl variables takes the form 4 :

$$
H_{D}=\frac{1}{2}\left(p^{2}+m_{0}^{2}\right)-\sum_{I=1}^{2} \frac{h}{2}(-1)^{I}\left(\mathfrak{z}_{I}^{A} p_{A \bar{B}} z_{I}^{\bar{B}}+\mathfrak{z}_{I}^{\bar{A}} p_{\bar{A} B} z_{I}^{B}\right)
$$

The Poisson algebra of the complex constraints can be easily calculated. From (10) and (11) it follows that:

$$
\left\{G_{I( \pm)}^{A}, G_{J( \pm)}^{B}\right\}=0, \quad\left\{G_{I(+)}^{A}, G_{J(-)}^{B}\right\}=4 \delta_{I J} m^{2}(p) \epsilon^{A B} .
$$

The functions (11) are, under the Poisson bracket, the mass-weighted eigenfunctions of (12):

$$
\left\{H_{D}, G_{I( \pm)}^{A}\right\}= \pm \frac{i h}{2}(-1)^{I} m(p) G_{I( \pm)}^{A} .
$$

As one should expect, the constraints $G_{I( \pm)}^{A}$ and $G_{I( \pm)}^{\bar{A}}$ are not independent:

$$
G_{I( \pm)}^{\bar{A}}=\mp \frac{i}{m(p)} p_{B}^{\bar{A}} G_{I( \pm)}^{B}
$$

Hence, according to (13) and (14), the systems $\left(H_{D}, G_{I( \pm)}^{A}\right)$ constitute polarized Poisson algebras of first class. From $\overline{\left(G_{I( \pm)}^{A}\right)}=G_{I(\mp)}^{\bar{A}}$ and (15) it follows that they are mutually complex conjugated.

\footnotetext{
${ }^{3}$ According to common convention $z_{I}^{\bar{A}}=\overline{\left(z_{I}^{A}\right)}$.

${ }^{4}$ All indices are raised and lowered by $\mathbf{S L}(2 ; \mathbb{C})$ invariant tensors $\epsilon^{A B}\left(\epsilon^{\bar{A} \bar{B}}\right)$ and their inverses $\epsilon_{A B}$ $\left(\epsilon_{\bar{A} \bar{B}}\right)$ respectively.
} 


\section{The quantum model}

\subsection{The space of states}

The Hilbert space of states corresponding to the classical system under consideration, consists in square integrable functions of space-time momentum $\left(p^{\mu}\right)$ and Weyl spinor coordinates $\left(z_{I}^{A}, z_{I}^{\bar{A}}\right)$. In this representation space the canonically conjugated variables are realized as the following operators: $\hat{x}^{\mu}=-i \partial / \partial p_{\mu}$ and $\hat{\mathfrak{z}}_{I}^{A}=i \epsilon^{A B} \partial / \partial z_{I}^{B}, \hat{\mathfrak{z}}_{I}^{\bar{A}}=$ $i \epsilon^{\bar{A} \bar{B}} \partial / \partial z_{I}^{\bar{B}}$.

It should be stressed that according to the conditions imposed already at the classical level, the momenta $\left(p^{\mu}\right)$ are restricted to the open domain $p^{2}<0$ supporting real massive particles. Since this domain is the union of two disjoint cone interiors $p^{0}>0$ and $p^{0}<0$, the Hilbert space of states decomposes into the corresponding direct sum:

$$
H=H^{\uparrow} \oplus H^{\downarrow} .
$$

The above direct components contain the functions of the future-pointed and pastpointed momenta respectively.

In the representation space above the operators corresponding to the spinorial constraints (11) are expressed by:

$$
G_{I( \pm)}^{A}=i p^{A \bar{B}} \frac{\partial}{\partial z_{I}^{\bar{B}}} \mp m(p) \epsilon^{A B} \frac{\partial}{\partial z_{I}^{B}}+p_{\bar{B}}^{A} z_{I}^{\bar{B}} \pm i m(p) z_{I}^{A},
$$

while the Dirac Hamiltonian (12) takes the following operator form:

$$
H_{D}=\frac{1}{2}\left(p^{2}+m_{0}^{2}\right)-\frac{i h}{2} \sum_{I=1}^{2}(-1)^{I}\left(z_{I}^{\bar{B}} p_{\bar{B}}{ }^{A} \frac{\partial}{\partial z_{I}^{A}}+z_{I}^{B} p_{B}{ }^{\bar{A}} \frac{\partial}{\partial z_{I}^{\bar{A}}}\right) .
$$

In order to analyze the spectrum of the model it is useful to distinguish the differential part of (18):

$$
S=-\frac{i h}{2} \sum_{I=1}^{2}(-1)^{I}\left(z_{I}^{\bar{B}} p_{\bar{B}}^{A} \frac{\partial}{\partial z_{I}^{A}}+z_{I}^{B} p_{B}{ }^{\bar{A}} \frac{\partial}{\partial z_{I}^{\bar{A}}}\right) .
$$

As it will appear clear the operator (19) is responsible for spin-mass correlation in the spectrum of the model. For this reason it will be called the spin-mass coupling operator.

The space of physical states of the model is defined as a kernel of either $G_{I(+)}^{A}$ or $G_{I(-)}^{A}$ constraints [14] (off-shell physical states) and $H_{D}$ kinematical constraint.

According to [15] and the detailed analysis of the paper [9] the physical states should be looked for within the vectors of the form:

$$
\Psi_{( \pm)}\left(p, z_{I}, \bar{z}_{I}\right)=W\left(z_{I}, \bar{z}_{I}\right) \Omega_{( \pm)}(p)
$$

where $W\left(z_{I}, \bar{z}_{I}\right)$ are the polynomials in the Weyl variables with momentum dependent coefficients.

The space (16) of the functions (20) splits in a natural way into the sum of the direct integrals:

$$
H^{\uparrow / \downarrow}=\int_{V_{( \pm)}} d^{4} p H^{\uparrow / \downarrow}(p)
$$


of the Hilbert spaces $H^{\uparrow}(p)$ and $H^{\downarrow}(p)$ localized at the momentum $p$, contained in $V_{( \pm)}$ - the future-pointed and the past-pointed cone interiors respectively.

The states $\Omega_{( \pm)}(p)$ (the spin vacua) are the Gaussian solutions of spinorial constraints $G_{I( \pm)}^{A} \Omega_{( \pm)}(p)=0$. It is easy to check that (up to multiplicative factors) they are given by:

$$
\Omega_{( \pm)}(p)=\exp \left( \pm \sum_{I=1}^{2} \frac{z_{I}^{\bar{A}} p_{\bar{A} B} z_{I}^{B}}{m(p)}\right)
$$

According to the adopted conventions the momentum matrix $\left(p_{\bar{A} B}\right)$ is negatively defined in the future-pointed $\left(p^{0}>0\right)$ light-cone interior and it is positive in past-pointed $\left(p^{0}<0\right)$ domain. For this reason one should take $\Omega_{(+)}(p)$ spin vacuum in $H^{\uparrow}(p)$ and $\Omega_{(-)}(p)$ state in $H^{\downarrow}(p)$. This choice guarantees that the states (20) belong to localized Hilbert space of the quantum model, i.e. they are square integrable with respect to Weyl variables. It also means, that any state from $H^{\uparrow / \downarrow}(p)$ can be represented as a superposition of the following vectors:

$$
\begin{aligned}
\Psi_{\left(j_{1}, j_{2}\right)}^{\uparrow / \downarrow}\left(p, z_{I}, \bar{z}_{I}\right)= & \sum_{n_{1}=0}^{2 j_{1}} \sum_{n_{2}=0}^{2 j_{2}} \Psi_{A_{1}^{1} \ldots A_{2 j_{1}-n_{1}}^{1} \bar{B}_{1}^{1} \ldots \bar{B}_{n_{1}}^{1} ; A_{1}^{2} \ldots A_{2 j_{2}-n_{2}}^{2} \bar{B}_{1}^{2} \ldots \bar{B}_{n_{2}}^{2}}(p) \cdot \\
& z_{1}^{A_{1}^{1}} \cdots z_{1}^{A_{2 j_{1}-n_{1}}^{1}} z_{1}^{\bar{B}_{1}^{1}} \cdots z_{1}^{{ }^{1}} z_{2}^{1} \cdots z_{2}^{A_{2 j_{2}-n_{2}}^{2}} z_{2}^{\bar{B}_{1}^{2}} \cdots z_{2}^{\bar{B}_{n_{2}}^{2}} . \\
& \cdot \Omega_{( \pm)}(p),
\end{aligned}
$$

of fixed integral degrees $\left(2 j_{1}, 2 j_{2}\right)$ in $\left(z_{1}^{A}, z_{1}^{\bar{A}}\right)$ and $\left(z_{2}^{A}, z_{2}^{\bar{A}}\right)$ complex variables respectively.

In the light of the above considerations one should impose $G_{I(+)}^{A}$ constraints in $H^{\uparrow}$ and $G_{I(-)}^{A}$ in $H^{\downarrow}$. Hence, the space $\widehat{H}$ of physical off-shell states is necessarily of the form of the following direct sum:

$$
\widehat{H}=\widehat{H}_{(+)}^{\uparrow} \oplus \widehat{H}_{(-)}^{\downarrow},
$$

where $\widehat{H}_{( \pm)}^{\uparrow / \downarrow}$ are the kernels of $G_{I( \pm)}^{A}$ operators correspondingly.

In order to find these kernels one may follow the direct method presented in the paper [9]. They are however much more transparently described in terms of spectrum generating algebra. This algebra contains the constraints operators (17) and in addition the following ones:

$$
D_{I( \pm)}^{A}=i p^{A \bar{B}} \frac{\partial}{\partial z_{I}^{\bar{B}}} \pm m(p) \epsilon^{A B} \frac{\partial}{\partial z_{I}^{B}}-p_{\bar{B}_{I}^{A}}^{A \bar{B}} \pm i m(p) z_{I}^{A}
$$

The above operators commute with these of (17). The only non-zero commutators are given by:

$$
\left[G_{I(+)}^{A}, G_{J(-)}^{\bar{B}}\right]=4 \delta_{I J} m(p) p^{A \bar{B}} \quad, \quad\left[D_{I(+)}^{A}, D_{J(-)}^{\bar{B}}\right]=4 \delta_{I J} m(p) p^{A \bar{B}} .
$$

Since one has $\left(G_{I( \pm)}^{A}\right)^{*}=G_{I(\mp)}^{\bar{A}}$ and $\left(D_{I( \pm)}^{A}\right)^{*}=D_{I(\mp)}^{\bar{A}}$, the operators (17) and (25) may serve as creation and anihilation operators in the space (21).

From the form of the generators of $\mathbf{S L}(2 ; \mathbb{C})$ transformations:

$$
L^{\mu \nu}=i\left(p^{\mu} \frac{\partial}{\partial p^{\nu}}-p^{\nu} \frac{\partial}{\partial p^{\mu}}\right)+\frac{i}{2} \sum_{I=1}^{2}\left(z_{I}^{A} \sigma_{A}^{(\mu \nu) B} \frac{\partial}{\partial z_{I}^{B}}+z_{I}^{\bar{A}} \sigma_{\bar{A}}^{(\mu \nu) \bar{B}} \frac{\partial}{\partial z_{I}^{\bar{B}}}\right)
$$

\footnotetext{
${ }^{5}$ They can be obtained as operator counterparts of the corresponding Noether conserved quantities calculated from (1) and (4).
} 
it follows that the constraints $G_{I( \pm)}^{A}$ and the operators $D_{I( \pm)}^{A}$ are of spinorial character and they carry spin $\frac{1}{2}$ :

$$
\left[L^{\mu \nu}, G_{I( \pm)}^{A}\right]=\frac{i}{2} G_{I( \pm)}^{B} \sigma_{B}^{(\mu \nu) A} \quad, \quad\left[L^{\mu \nu}, D_{I( \pm)}^{A}\right]=\frac{i}{2} D_{I( \pm)}^{B} \sigma_{B}^{(\mu \nu) A}
$$

while the kinematic constraint:

$$
H_{D}=\frac{1}{2}\left(p^{2}+m_{0}^{2}\right)-\frac{h}{8 m^{2}(p)} \sum_{I=1}^{2}(-1)^{I}\left(G_{I(+)}^{A} p_{A \bar{B}} G_{I(-)}^{\bar{B}}-D_{I(+)}^{A} p_{A \bar{B}} D_{I(-)}^{\bar{B}}\right)
$$

is scalar.

One may directly check that:

$$
G_{I( \pm)}^{A} \Omega_{( \pm)}(p)=D_{I( \pm)}^{A} \Omega_{( \pm)}(p)=0
$$

and consequently, any state (23) from the space $H^{\uparrow / \downarrow}(p)$ can be equivalently and more transparently expressed in the Fock-type representation:

$$
\begin{aligned}
& \Psi_{\left(j_{1}, j_{2}\right)}^{\uparrow / \downarrow}(p)=\sum_{n_{1}=0}^{2 j_{1}} \sum_{n_{2}=0}^{2 j_{2}} \Phi(p)_{A_{1} . . A_{2 j_{1}-n_{1}} B_{1} . . B_{n_{1}} C_{1} . . C_{2 j_{2}-n_{2}} D_{1} . . D_{n_{2}}(p)} \cdot \\
& \cdot D_{1(\mp)}^{A_{1}} . . D_{1(\mp)}^{A_{2 j_{1}-n_{1}}} G_{1(\mp)}^{B_{1}} . . G_{1(\mp)}^{B_{n_{1}}} D_{2(\mp)}^{C_{1}} . . D_{2(\mp)}^{C_{2 j_{2}-n_{2}}} G_{2(\mp)}^{D_{1}} . . G_{2(\mp)}^{D_{n_{2}} \Omega_{( \pm)}(p) .}
\end{aligned}
$$

As one has $\left[G_{I(+)}^{A}, G_{J(-)}^{B}\right]=4 i \delta_{I J} m^{2}(p) \epsilon^{A B}$ and $\left[D_{I(+)}^{A}, D_{J(-)}^{B}\right]=4 i \delta_{I J} m^{2}(p) \epsilon^{A B}$, the constraints are easily solvable now. They simply mean that $G_{I(\mp)}^{A}$ excitations should be absent in (31) and for the solutions one gets:

$$
\Psi_{\left(j_{1}, j_{2}\right)}^{\uparrow / \downarrow}(p)=\Phi(p)_{A_{1} . . A_{2 j_{1}} C_{1} . . C_{2 j_{2}}} D_{1(\mp)}^{A_{1}} . . D_{1(\mp)}^{A_{2 j_{1}}} D_{2(\mp)}^{C_{1}} . . D_{2(\mp)}^{C_{2 j_{2}}} \Omega_{( \pm)}(p) .
$$

It is worth to mention that the constraints $G_{I( \pm)}^{A}$ imposed on the states in the form of (23) become the Dirac-type equations for the coefficients of the polynomials. In the case of $\widehat{H}_{(+)}^{\uparrow}(p)$ they take the following form:

$$
\begin{aligned}
& -\left(n_{I}+1\right) p_{A_{2 j_{I}-n_{I}}^{I}}^{\bar{B}_{n_{I}+1}^{I}} \Psi_{A_{1}^{1} \ldots A_{2 j_{1}-n_{1}-\delta_{I, 1}}^{1} \bar{B}_{1}^{1} \ldots \bar{B}_{n_{1}+\delta_{I, 1}}^{1} ; A_{1}^{2} \ldots A_{2 j_{2}-n_{2}-\delta_{I, 2}}^{2} \bar{B}_{1}^{2} \ldots \bar{B}_{n_{2}+\delta_{I, 2}}^{2}}(p)= \\
& =i m(p)\left(2 j_{I}-n_{I}\right) \Psi_{A_{1}^{1} \ldots A_{2 j_{1}-n_{1}}^{1} \bar{B}_{1}^{1} \ldots \bar{B}_{n_{1}}^{1} ; A_{1}^{2} \ldots A_{2 j_{2}-n_{2}}^{2} \bar{B}_{1}^{2} \ldots \bar{B}_{n_{2}}^{2}}(p) .
\end{aligned}
$$

where $I=1,2$ and $n_{I}=0, \ldots, 2 j_{I}-1$.

In the complementary space $\widehat{H}_{(-)}^{\downarrow}(p)$ of anti-particles the above equations are completely analogous.

In order to find the spectrum of the model one should impose the kinematical constraint $H_{D}(29)$ on the solutions of (32). This constraint correlates the masses of the particles with their spins. The detailed analysis of the spectrum will be given in the next subsection. 


\subsection{Spin and mass spectrum}

In order to find the spectrum explicitly it is convenient to use the following decomposition of the off-shell spaces:

$$
\widehat{H}_{(+)}^{\uparrow}(p)=\bigoplus_{\left(j_{1}, j_{2}\right)} \widehat{H}_{(+)}^{\uparrow\left(j_{1}, j_{2}\right)}(p)
$$

where $\widehat{H}_{(+)}^{\uparrow\left(j_{1}, j_{2}\right)}(p)$ contains the polynomials of fixed bidegree $\left(2 j_{1}, 2 j_{2}\right)$ of the form of (32). From (29) and commutations relations (26) it follows that the spin-mass coupling operator $S$ of (19) is diagonal and acts in the following way:

$$
S \widehat{H}_{(+)}^{\uparrow\left(j_{1}, j_{2}\right)}(p)=h m(p)\left(j_{1}-j_{2}\right) \widehat{H}_{(+)}^{\uparrow\left(j_{1}, j_{2}\right)}(p) .
$$

Hence, the kinetic constraint imposes the following mass-shell condition on the particle momenta:

$$
\frac{1}{2}\left(p^{2}+m_{0}^{2}\right)+h m(p)\left(j_{1}-j_{2}\right)=0,
$$

with the unique solution leading to normalizable states, given by:

$$
m_{\left(j_{1}, j_{2}\right)}^{\uparrow}=\sqrt{h^{2}\left(j_{1}-j_{2}\right)^{2}+m_{0}^{2}}+h\left(j_{1}-j_{2}\right) ; j_{1}, j_{2} \geq 0 .
$$

One may analogously find the masses of the states supported by the past-pointed cone interior:

$$
m_{\left(j_{1}, j_{2}\right)}^{\downarrow}=\sqrt{h^{2}\left(j_{1}-j_{2}\right)^{2}+m_{0}^{2}}-h\left(j_{1}-j_{2}\right) ; j_{1}, j_{2} \geq 0 .
$$

According to (37) and (38), for on-shell physical states, the direct integrals corresponding to (21) are restricted to the respective mass-shells. The spaces of physical on-shell states will be denoted by $\widetilde{H}_{(+)}^{\uparrow}$ and $\widetilde{H}_{(-)}^{\downarrow}$ respectively.

The comparison of the formulae (37) and (38) clearly indicates that the spectrum of the model is CPT invariant[10], i.e. it is symmetric with respect to particle-antiparticle exchange. This property was missing in the simple prototype model presented in [9].

In order to perform a more detailed analysis of the spectrum it is useful to distinguish two families of subspaces of the on-shell space $\widetilde{H}_{(+)}^{\uparrow}(p)$, namely:

$$
\widetilde{H}_{(+)}^{\uparrow\left(j_{1}, 0\right)}(p) \quad \text { and } \quad \widetilde{H}_{(+)}^{\uparrow\left(0, j_{2}\right)}(p)
$$

where the numbers $j_{1}$ and $j_{2}$ have direct physical interpretation as particle spins [15]. As it is clearly visible from (32) these spaces describe the states of single particles with spins $j_{1}$ and $j_{2}$, and with the corresponding masses:

$$
m_{j_{1}}^{\uparrow}=\sqrt{h^{2} j_{1}^{2}+m_{0}^{2}}+h j_{1}, m_{j_{2}}^{\uparrow}=\sqrt{h^{2} j_{2}^{2}+m_{0}^{2}}-h j_{2} .
$$

The antiparticles of $\widetilde{H}_{(+)}^{\uparrow\left(j_{1}, 0\right)}(p)$ and $\widetilde{H}_{(+)}^{\uparrow\left(0, j_{2}\right)}(p)$ are represented in the spaces:

$$
\widetilde{H}_{(-)}^{\downarrow\left(0, j_{1}\right)}(p) \quad \text { and } \quad \widetilde{H}_{(-)}^{\downarrow\left(j_{2}, 0\right)}(p)
$$

\footnotetext{
${ }^{6}$ The second, negative root of (37) has to be rejected because it makes the exponent (22) growing very fast at infinity.
} 
respectively.

According to (21), as it was already mentioned, the localized spaces (39) and (41) fit together into direct integrals over the corresponding mass-shells $S_{+}\left(j_{I}\right)$ :

$$
\widetilde{H}_{(+)}^{\uparrow\left(j_{1}, 0\right)}=\int_{S_{+}\left(j_{1}\right)} d \mu_{j_{1}}(p) \widetilde{H}_{(+)}^{\uparrow\left(j_{1}, 0\right)}(p) \quad \text { and } \quad \widetilde{H}_{(+)}^{\uparrow\left(0, j_{2}\right)}=\int_{S_{+}\left(j_{2}\right)} d \mu_{j_{2}}(p) \widetilde{H}_{(+)}^{\uparrow\left(0, j_{2}\right)}(p),
$$

with respect to Lorentz invariant measures $d \mu_{j_{I}}$.

Together with their antiparticles partners they describe two particle-antiparticle pairs located at two diverging Regge trajectories. Hence, the corresponding mass-levels are non-degenerate as in the case of the prototype model of [9]. For this reason these trajectories will be called elementary. They are illustrated at Fig.1A 7 .

Besides of the particle pairs located at the elementary trajectories, there is a family of Hilbert spaces containing the polynomials non-zero degree in both, $\left(z_{1}^{A}, z_{1}^{\bar{A}}\right)$ and $\left(z_{2}^{A}, z_{2}^{\bar{A}}\right)$ Weyl variables, i.e. of the spaces:

$$
\widetilde{H}_{(+)}^{\uparrow\left(j_{1}, j_{2}\right)}(p) \quad \text { where } \quad j_{I}>0 ; I=1,2 .
$$

The momenta of the states from (43) are obviously constrained by mass-shell condition (37). The corresponding antiparticles are contained in $\widetilde{H}_{(-)}^{\downarrow\left(j_{2}, j_{1}\right)}(p)$ and according to (38) they have exactly this same masses.

In contrast to $\widetilde{H}_{(+)}^{\uparrow\left(j_{1}, 0\right)}$ and $\widetilde{H}_{(+)}^{\uparrow\left(0, j_{2}\right)}$ the spaces (43) are reducible with respect to $\mathbf{S L}(2 ; \mathbb{C})$ group representation, i.e. they decay into direct sums of the subspaces containing the states with fixed spins, i.e. those of elementary particles. This property is clearly visible from the form of the solution of $G_{I(+)}^{A}$ constraints (17) which is explicitly given in (32). For this reason it is natural to call the states from (43) the clusters.

The spin content of the spaces $\widetilde{H}_{(+)}^{\uparrow\left(j_{1}, j_{2}\right)}(p)$ at fixed momentum can be obtained by the standard simple rule of the decomposition of $\mathbf{S U}(2)$ (the little group of massive momentum $p$ ) representations [16]:

$$
\widetilde{H}_{(+)}^{\uparrow\left(j_{1}, j_{2}\right)}(p)=\bigoplus_{j=\left|j_{1}-j_{2}\right|}^{j_{1}+j_{2}} \widetilde{H}_{(+)}^{\uparrow j}(p)
$$

where $\widetilde{H}_{(+)}^{\uparrow j}(p)$ denote the irreducible subspace of spin $j$.

From (44) it is clear that keeping the mass of the particle fixed (fixed value of the difference $j_{1}-j_{2}$ ) one may obtain arbitrarily high spin states. Hence, the states from $\widetilde{H}_{(+)}^{\uparrow\left(j_{1}, j_{2}\right)}(p)$ generate infinite spin degeneracy of the mass levels. The corresponding cluster trajectories are illustrated on Fig.1B. From this diagram it is clear that the cluster states contribute to elementary trajectories and in addition, they generate their own ones.

\section{Conclusions and outlook}

As it was shown the model considered in this paper has CPT invariant mass spectrum. It consists of elementary (non-degenerate) Regge trajectories and highly degenerate

\footnotetext{
${ }^{7}$ For $p^{0}<0$ one obtains exactly the same trajectories of antiparticles.
} 

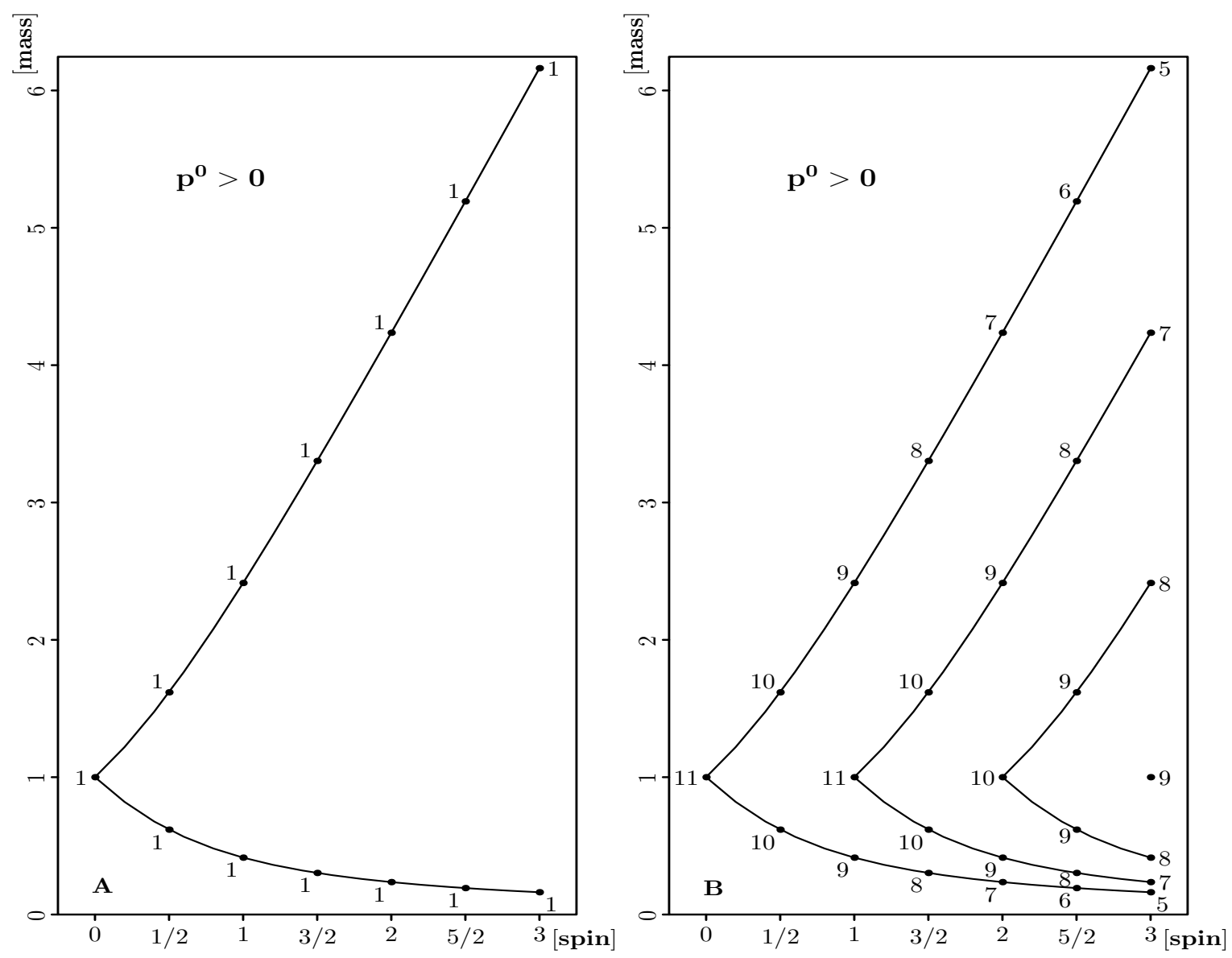

Figure 1: A - The elementary trajectories for particles (in $m_{0}$ units). B - The degeneracy of the spectrum of clusters (in $m_{0}$ units and with $j_{I}$ ranging from 0 to $11 / 2$ ).

sea of cluster particles.

According to the considerations of second chapter, in the case of elementary trajectories, the solution (32) describes the wave function of the one elementary particle with fixed spin (in the sense of $\mathbf{S L}(2 ; \mathbb{C})$ representations). The particle is accompanied by the corresponding antiparticle.

In the case of cluster (44) the wave functions solving the constraints equations (17), (18) do not represent the elementary objects, but the families of particles with spins ranging from $\left|j_{1}-j_{2}\right|$ to $j_{1}+j_{2}$. The solution (32) together with mass-shell condition describe an ensemble of particles.

These objects make an impression as being untypical, however the idea of cluster fields (in that case with respect to mass not spin) was proposed and developed almost 50 years ago in the series of papers [25].

The analysis at the classical level indicates that the infinite degeneracy of the mass levels might appear as an effect of the substraction of the interaction term (4) from (11), which makes the classical dynamics of the corresponding spinors linear, and almost completely independent. This substraction results in the independence of the polarized constraints (11) for both spinors and simple form of Dirac Hamiltonian. For this very 
reason, the corresponding wave functions contain products of independent polynomials in both independent Weyl spinors, which in turn amounts to the decomposition given in (44).

The detailed analysis of the model described by (1), i.e. containing the non-linear interaction (4) is already in progress, and will be presented in the future paper.

\section{Acknowledgements}

One of the authors (M.D.) would like to thank the friends and colleagues from the Institute of Theoretical Physics in Białystok for warm hospitality.

The authors would like to thank also prof. J.Lukierski, prof. Z.Haba and dr. A.Błaut for interesting discussions.

Special thanks (from Z.H.) are due to Bożena Gonciarz for her inspiration.

This work is partially supported by KBN grant 1P03B01828.

\section{References}

[1] A.O.Barut, N.Zoughi, Phys. Rev. Lett. 52 (1984) 2009

[2] V.D.Gershun, V.I.Tkach, JETP Lett. (1979) 320

[3] P.S.Howe, S.Penati, M.Pernici, P.Townsend, Phys. Lett. B215 (1988) 255

[4] S.Fedoruk, J.Lukierski, Phys. Lett. B632 (2006) 371-378

[5] A.Bette, J.de Azcarraga, J.Lukierski, C.Miquel-Espanya, Phys. Lett. B595 (2004)

[6] A.Frydryszak, Lagrangian models of particles with spin, Published in From field theory to quantum groups, Singapore World Scientific Publishing (1996)

[7] S.M.Kuzenko, S.L.Lyakhovich, A.Yu.Segal, Int. J. Mod. Phys. A10 (1995) 1529

[8] Z.Hasiewicz, F.Defever, P.Siemion, Int. J. Mod. Phys. A 7 (1992) 3979-3996

[9] M.Daszkiewicz, Z.Hasiewicz, C.Walczyk, High spin particles with spin-mass coupling, Advances in Applied Clifford Algebras (in press)

[10] R.F.Streater, A.S.Wightman PCT Spin - Statistic and all that Benjamin, New York 1964

R.Jost The General Theory of Quantized Fields AMS, Providence, Rhode Island 1965

[11] E.Schroedinger, Sitzugsh. Preuss. Akad. Wiss. Phys.-Math. Kl. 24 (1930) 418; 3 (1931) 1

[12] A.O.Barut, A.J.Bracken, Phys. Rev. D23 (1981) 2454

[13] M.Rivas, Classical elementary particles, spin, zitterbewegung and all that physics/0312107 
[14] S.Gupta, Proc. Roy. Soc. A63 (1950) 681

K.Bleuler, Helv. Phys. Acta 23 (1950) 567

[15] Jan Łopuszański Spinor Calculus PWN Warsaw 1985 (in Polish) A.O.Barut, R.Rączka Theory of Group Representations and Applications PWN Warsaw 1977

[16] Jan Mozrzymas Application of Group Theory in Physics PWN Warsaw 1976 (in Polish)

M.Hamermesh Group Theory PWN Warsaw 1968

[17] A.R.Swift, Phys. Rev. 176 (1968) 1848-1855

[18] W.J.Zakrzewski, Nuovo Cim. A60 (1969) 263-290

[19] A.R.Swift, R.W.Tucker, Nuovo Cim. A67 (1970) 345-355

[20] A.R.Swift, R.W.Tucker, Phys. Rev. D1 (1970) 2894-2900

[21] C.Rebbi, Phys. Rept. 12 (1974) 1-73

[22] H.Hata, K.Itoh, T.Kugo, H.Kunitomo, K.Ogawa, Phys. Rev. D34 (1986) 2360

[23] E.Witten, Nucl. Phys. B268 (1986) 253

[24] A.M.Polyakov, Phys. Lett. B103 (1981) 207

[25] O.W.Greenberg, Ann. of Phys. 16 (1961) 158

A.L.Licht, Ann. of Phys. 34 (1965) 161

L.Turko, Nucl. Phys. B114 (1976) 535-545 